TRANSACTIONS OF THE

AMERICAN MATHEMATICAL SOCIETY

Volume 349, Number 7, July 1997, Pages 2737-2754

S 0002-9947(97)01873-4

\title{
SPECIFICATION ON THE INTERVAL
}

\author{
JÉRÔME BUZZI
}

\begin{abstract}
We study the consequences of discontinuities on the specification property for interval maps. After giving a necessary and sufficient condition for a piecewise monotonic, piecewise continuous map to have this property, we show that for a large and natural class of families of such maps (including the $\beta$-transformations), the set of parameters for which the specification property holds, though dense, has zero Lebesgue measure.

Thus, regarding the specification property, the general case is at the opposite of the continuous case solved by A.M. Blokh (Russian Math. Surveys 38 (1983), 133-134) (for which we give a proof).
\end{abstract}

\section{INTRODUCTION}

R. Bowen [5] has introduced the following notion. Given a self-map on a compact metric space $f: X \rightarrow X$, one says that $f$ has the specification property, if, for every $\epsilon>0$, there exists a constant $D<\infty$ such that, given $\left(x_{1}, l_{1}\right), \ldots,\left(x_{r}, l_{r}\right) \in$ $X \times \mathbb{N}, 0 \leq r<\infty$, if we let $L(s)=l_{1}+\cdots+l_{s-1}+(s-1) D$ for $1 \leq s \leq r+1$, then there exists $z \in X$ such that $f^{L(r+1)}(z)=z$ and $d\left(f^{L(s)+k}(z), f^{k}\left(x_{s}\right)\right)<\epsilon$ for $0 \leq k<l_{s}$ and $s=1, \ldots, r$.

In other words, given an arbitrary number of arbitrarily long segments of orbits, one can find a periodic orbit which follows each one of them, with a precision $\epsilon>0$, and "switch" from one segment to another in a fixed amount of time depending only on $\epsilon$.

This property is very strong. It allows the study of generic properties of invariant measures and, under the additional hypothesis of expansiveness, it implies that there exists a unique equilibrium measure for any smooth potential (see, e.g., [6]). It is so strong that one could wonder whether it is realized by many natural systems, except of course by subshifts of finite type and the like.

F. Hofbauer [10], using his Markov diagram, introduced previously in [7], has shown that all continuous piecewise monotonic maps have a weak specification property. This weak property implies the generic properties of invariant measures which we alluded to, but not the result about equilibrium states.

A.M. Blokh completely solved the problem on the interval in the continuous case, in quite an unexpected way:

Received by the editors September 12, 1995.

1991 Mathematics Subject Classification. Primary 58F03; Secondary 54H20.

Key words and phrases. One-dimensional dynamics, piecewise monotonic, piecewise continuous maps, specification property, Lebesgue measure in parameter space, symbolic dynamics, Hofbauer's Markov diagram.

(C) 1997 American Mathematical Society 
1.1 Theorem (A.M. Blokh [3]). Every continuous self-map of the interval has the specification property iff it is topologically mixing (i.e., for every pair of non-empty open sets $U, V, f^{n}(U) \cap V \neq \emptyset$ for all large $\left.n\right)$.

For the sake of completeness we give a short proof of this result of A.M. Blokh in Appendix A.

This paper addresses the following question, most natural after A.M. Blokh's result: what happens for general interval maps (i.e., if one allows discontinuities)? We are going to show that the situation completely changes: the specification property becomes exceptional. For instance:

1.2 Counterexample. Though, for all $\beta>1$, all maps $T_{\beta}:[0,1] \rightarrow[0,1]$ defined by:

$$
T_{\beta}: x \mapsto\{\beta x\} \quad(\{\cdot\} \in[0,1[: \text { fractional part })
$$

are topologically mixing, the set of $\beta>1$ such that the map $T_{\beta}$ has the specification property is dense, but has Lebesgue measure zero.

We prove this fact as a special case of a general result, the Main Theorem below (see the comments after the Main Theorem for generalizations). Now let us state the general result. First we give some definitions.

1.3 Definition. A p.m.m. (piecewise monotonic-continuous map) is a map $f$ : $[0,1] \backslash C(f) \rightarrow[0,1]$ with $C(f)$ a finite set, called the set of critical points, such that, writing $P$ for the set of the connected components of $[0,1] \backslash C(f)$ :

1. for every $U \in P, f: U \rightarrow f(U)$ is a homeomorphism;

2. no critical point $c \in C(f)$ is removable: $f$ cannot be extended to any neighbourhood $V$ of $c$ in such a way that $f: V \rightarrow f(V)$ is a homeomorphism.

$P$ is called the natural partition of $f$.

Recall that a function is $C^{r}$ with $r$ non integer iff it is $C^{k}$ with $k$ the largest integer smaller than $r$ and its $r$ th derivative is Hölder with exponent $r-k$.

1.4 Definition. Let $r \geq 0$. A $C^{r}$ family of p.m.m.'s is $\left\{f_{t}\right\}_{t \in T}$ such that $T=$ $\bigcup_{j=1}^{\infty} \overline{T_{j}}$ and each $T_{j}$ is an interval, for which there exists:

1. an integer $1 \leq N<\infty$.

2. $0<d_{1}(t)<\cdots<d_{N-1}(t)<1$ - critical points, assumed to be $C^{r}$ functions of $t$. We write $C\left(f_{t}\right)=\left\{d_{1}(t), \ldots, d_{N-1}(t)\right\}$.

3. $f_{i}: T \times[0,1] \rightarrow \mathbb{R}(i=1, \ldots, N)$ strictly monotonic mappings of class $C^{r}-$ the "pieces"

such that $f_{t}$ is defined from $[0,1] \backslash C\left(f_{t}\right)$ to $[0,1]$ and coincides with the p.m.m.:

$$
\begin{aligned}
& x \in[0,1] \backslash C\left(f_{t}\right) \mapsto f_{i}(t, x), \\
& \qquad i=i(x):=\max \left\{j \in\{1, \ldots, N\}: d_{j-1}(t)<x<d_{j}(t)\right\}
\end{aligned}
$$

(where we set, for convenience, $d_{0}(t)=0, d_{N}(t)=1$ ).

Notations. As a p.m.m. $f$ is not defined on the whole of $[0,1]$, so we make the following conventions. We write $f(S)$ for $f(S \backslash C(f)$ ). We write $g(x+)$ (resp. $g(x-)$ ) for the limit to the right (resp. to the left) of $x$ of the function $g$ (which can be $f_{t}$, $\partial_{u} f_{t}$ (with $u=x$ or $\left.t\right), f_{t}^{n}$, etc.). We call such an association of a point $x$ with a side + or - an endpoint. Often we shall write $x \pm, y \mp$, etc. Of particular interest to us will be the endpoints of the natural partition $P: c+, c-$ for $c \in C(f)$. 
In particular, we define in this way the virtual orbit under a p.m.m. $f$, starting from $x \pm$ as the sequence of limits $\left\{f^{k}(x \pm)\right\}_{k>0}$.

We remark that the union of all virtual orbits is countable so they have no effect on the properties of specification or topological mixing.

1.5 Main Theorem. Let $\{f\}_{t \in T}$ be a $C^{1+\theta}$ family of p.m.m.'s $(0<\theta<1)$. Assume that, for Lebesgue-almost all $t \in T, f_{t}$ is:

(A1) completely discontinuous (on the critical set): for every discontinuity $d_{i}(t)$, the left and right limits of $f_{t}$ at $d_{i}(t)$ are distinct.

(A2) non-degenerate: there exists an endpoint $e=e(t)\left(e(t)\right.$ standing for $\left.d_{i}(t) \pm\right)$ such that the sum:

$$
d_{i}^{\prime}(t)+\sum_{n \geq 1} \frac{\partial_{t} f_{t}\left(f_{t}^{n-1}(e)\right)}{\partial_{x} f_{t}^{n}(e)}
$$

does not converge to zero.

(A3) expanding:

$$
\inf _{x \in[0,1] \backslash C\left(f_{t}\right)}\left|\partial_{x} f_{t}(x)\right|>1
$$

Then the set $T_{\text {spec }}$ of values $t \in T$ such that $f_{t}$ has the specification property has Lebesgue measure zero.

On the other hand, the set $T_{\text {coll }}$ of values $t \in T$ such that critical orbits collide, i.e., $f_{t}^{n}(c \pm)=d$ for some $c, d \in C\left(f_{t}\right)$ and $n \geq 1$, is dense in $T$.

1.6 Corollary. Under the additional assumption:

(A4) there is essentially only one critical orbit, i.e., for all $t \in T$ :

$$
\begin{gathered}
f_{t}(0)=f_{t}\left(d_{1}(t)+\right)=\cdots=f_{t}\left(d_{N-1}(t)+\right)=0, \\
f_{t}\left(d_{1}(t)-\right)=\cdots=f_{t}\left(d_{N-1}(t)-\right)=1,
\end{gathered}
$$

the set $T_{\text {spec }}$ is dense in $T$.

Comments. 1. Statements similar to 1.2 can be deduced from our result for much more general families of p.m.m. than $\beta$-transformations: consider $x \mapsto\{\beta x+\alpha\}$ or more generally $x \mapsto\left\{f_{t}(x)\right\}$ with $f_{t}$ of class $C^{1+\theta}$ such that: $\partial_{t} f_{t}>0, \partial_{x} f_{t} \geq$ $\lambda(t)>1$. We call this class of maps the expanding maps modulo 1 . We have the same conclusions as in the case of $\beta$-transformations: $T_{\text {spec }}$ has Lebesgue measure zero and, if $f_{t}(0)=0, T_{\text {spec }}$ is dense.

2. Clearly, condition (A1) is satisfied by "almost all" p.m.m. in any reasonable sense. However we must stress that we do not know of any simple necessary and sufficient condition for the specification property (like Proposition 2.1 below) in the general case, i.e., if the p.m.m. has among its critical points both discontinuity and continuity points.

3. There has to be some condition like (A2) above so as to avoid fictitious dependence on $t$, such as in a family like:

$$
f_{t}(x)=\varphi_{t} \circ f \circ \varphi_{t}^{-1}
$$

where $\varphi_{t}:[0,1] \rightarrow[0,1]$ is a family of diffeomorphisms and $f$ any p.m.m.

It suggests the following question. Assume that a family is expanding (condition A3) and that the series in (A2) converges to zero for all $t \in T$. Does this imply that the family is of the form $\left({ }^{*}\right)$ above? 
4. P. Walters [12] (see F. Hofbauer and G. Keller [11] for a generalization) has shown that existence and uniqueness of the equilibrium measure with respect to a smooth potential (one of the key properties of systems with specification) hold for all $\beta$-transformations.

It is natural to ask whether this property can be deduced from a suitably weakened property of specification which would be satisfied by these transformations.

In section 2 we state a necessary and sufficient condition for the specification property under condition (A1). The proof of necessity is immediate. The proof of sufficiency is more complex. It uses Hofbauer's Markov diagram. In section 3, we prove the main theorem by using standard techniques. Section 4 checks that the family of $\beta$-transformations satisfies the hypothesis of the theorem.

\section{CONDITION FOR THE SPECIFICATION PROPERTY}

This section is devoted to the proof of the following criterium:

2.1 Proposition. Let $f:[0,1] \backslash C(f) \rightarrow[0,1]$ be a p.m.m. Assume that it is completely discontinuous.

$f$ has the specification property if and only if the following two conditions hold:

(S1) $f$ is topologically mixing.

(S2) orbits of discontinuities do not get too close to discontinuities on the "wrong side", i.e., for every discontinuity $d \pm$, for all $n \geq 1$ :

$$
\left|f^{n}(d \pm)-e\right| \geq \alpha>0 \quad \text { with } \alpha \text { some constant }
$$

if $e$ is any discontinuity, distinct from $f^{n}(d \pm)$, such that $e$ and $f^{n}(] d, d \pm \varepsilon[)$ are on the same side of $f^{n}(d \pm)$ (for $\epsilon>0$ small enough).

Remark. After this paper was written, I was told of an article by A. BertrandMathis [2, Theorem II] about $\beta$-shifts, the symbolic dynamical systems associated to $\beta$-transformations. This article contains a necessary and sufficient condition for the specification property among these particular systems in terms of the so-called symbolic $\beta$-expansion of the critical point. It is easily seen to be equivalent to the above proposition restricted to $\beta$-shifts.

Proof of Necessity. Clearly specification implies topological mixing so that (S1) is necessary. Assume that (S2) is not fulfilled: for some discontinuities $d \pm, e$, there exists a sequence of integers $n_{i}$ such that: $\left|f^{n_{i}}(d \pm)-e\right|$ decreases to zero, $e$ being on the wrong side.

The Bowen ball of radius $\epsilon>0$ and order $n \geq 1$ centered at $x \in[0,1]$ is:

$$
B_{n}(x, \epsilon)=\left\{y \in[0,1]:\left|f^{k}(y)-f^{k}(x)\right|<\epsilon \text { for } k=0, \ldots, n-1\right\} .
$$

We define the Bowen ball centered at $x \pm$ in the obvious way, by replacing $f^{k}(x)$ by the limit $f^{k}(x \pm)$.

2.2 Lemma. If $f:[0,1] \rightarrow[0,1]$ is an arbitrary p.m.m. then, for $\epsilon>0$ small enough, for any $x \in[0,1]$, any $n \geq 0$, we have:

$$
f^{k} B_{n}(x, \epsilon) \text { is an interval } \quad(0 \leq k<n-1) .
$$

More precisely, the restrictions $f^{k} \mid B_{n}(x, \epsilon)$ are continuous.

If $f$ is a completely discontinuous p.m.m., then these restrictions are homeomorphisms. 
The lemma is obvious: it is enough to take $\epsilon>0$ so small that, for every discontinuity point $d$, we have that $f(] d-\epsilon, d[)$ and $f(] d, d+\epsilon[)$ are separated by a distance at least $\epsilon>0$. The lemma implies that $\left.f^{n_{i}} B_{n_{i}+2}(d \pm, \epsilon) \subset\right] f^{n_{i}}(d \pm), e[$. Hence the images of these Bowen balls have arbitrarily small diameter.

Now, the specification property would imply that, for arbitrarily small $\epsilon>0$, one can go from $f^{n} B_{n}(x, \epsilon)$ to any Bowen ball of radius $\epsilon$ in a "switching time" $M$ depending only on $\epsilon$. In particular $f^{M}\left(f^{n} B_{n}(x, \epsilon)\right)$ should be $\epsilon$-dense. We claim that, for $\epsilon<1 / 4$, this implies that $\operatorname{diam}\left(f^{n} B_{n}(x, \epsilon)\right)$ is not too small, contradicting what we have just seen. Let us prove the claim. Fix $M$. Let $I$ be an interval with small diameter $\operatorname{diam}(I)$ (i.e., $\operatorname{diam}(I)$ is smaller than some positive function of $M$ ). Let $n_{1}=\min \left\{k \geq 0: f^{k}(I) \cap C(f) \neq \emptyset\right\}$. If $n_{1} \geq M$ then $f^{M} \mid I$ is continuous and therefore $f^{M}(I)$ is an interval with small diameter and is not $\epsilon$-dense. Assume $n_{1}<M$.

$f^{n_{1}}(I)$ is small by continuity so it cannot contain more than one critical point. Let us split $f^{n_{1}}(I)$ into $I_{1} \cup\{c\} \cup I_{2}, c$ being the critical point met by $f^{n_{1}}(I)$. Let $n_{2}=\min \left\{k \geq 0: f^{k}\left(I_{1}\right) \cap C(f) \neq \emptyset\right\}$. If $n_{2}<M$ then $f^{n_{2}}\left(I_{1}\right)$ is small so that $f^{n_{2}}(c-)$ is close to, but distinct from a discontinuity. This implies that $n_{2}$ is large, contradicting the hypothesis $n_{2}<M$. Hence $n_{2} \geq M$.

We have shown that $f^{M} \mid I_{1}$ is continuous. It is the same for $f^{M} \mid I_{2}$. Hence, for $I$ of diameter small enough (with respect to $M$ ), $f^{M}(I)$ is either a small interval or the union of two small intervals $f^{M-n_{1}}\left(I_{1}\right), f^{M-n_{1}}\left(I_{2}\right)$ : it cannot be $\epsilon$-dense in $[0,1]$ for $\epsilon<1 / 4$. The claim, and therefore the necessity of (S1)-(S2), is proved.

We remark that the necessity of conditions (S1)-(S2) is enough to prove the part "zero Lebesgue measure" of the Main Theorem.

Preliminaries to the Proof of Sufficiency. Let $f$ be a p.m.m. completely discontinuous and satisfying (S1)-(S2). To prove the specification property we will study the symbolic dynamics of $f$.

Recall that $P$ is the natural partition of $f$. The itinerary of a point $x$ is the unique sequence $\iota(x) \in P^{\mathbb{N}}$ defined by: $A=\iota(x)$ iff $f^{k}(x) \in A_{k}$. It is defined for $x \in[0,1] \backslash C^{-}(f)$ with $C^{-}(f)=\bigcup_{k>0} f^{-k} C(f)$. An endpoint $x \pm(x \in[0,1])$ defines a unique itinerary naturally associated with the virtual orbit starting from $x \pm: \iota(x \pm)=A$ iff $f^{k}(x \pm h) \in A_{k}$ for $h>0$ small enough.

A finite itinerary is a finite sequence coinciding with the beginning of some $\iota(x)$. The set of points whose itineraries coincide with the itinerary of $x$ up to and including time $n-1$ is written $H_{n}(x)$. It is called the $n$-homterval containing $x$ as it is the maximal interval $J$ containing $x$ such that $f\left|J, \ldots, f^{n}\right| J$ are homeomorphisms on their images. $H_{n}(x \pm)$ is defined to be $H_{n}(x \pm h)$ for small $h>0$.

A homterval is a non-trivial interval on which $f^{n}$ is a homeomorphism for all $n \geq 0$. If $J$ is a maximal homterval, then there are two possibilities: (i) for some $n \geq 1, f^{n}$ maps $J$ homeomorphically into itself: every point of $J$ converges to some fixed point of $f^{2 n}$; or (ii) the intervals $f^{n}(J)$ are disjoint from the interior of $J$. Both possibilities contradict topological mixing.

Therefore our map $f$ has no homtervals.

Remark that this is equivalent to say that given an infinite sequence $A \in P^{\mathbb{N}}$ there exists at most one point or virtual orbit the itinerary of which is $A$. It implies, in particular, that if a point $x$ has a periodic itinerary, then the orbit of $x$ is also periodic, with the same period. Under our assumptions, the Bowen balls are very simply controlled in terms of symbolic dynamics: 
2.3 Lemma. Let $f:[0,1] \backslash C(f) \rightarrow[0,1]$ be a p.m.m.

$\left(^{*}\right)$ If $f$ has no homtervals, then, for every $\epsilon>0$, there exists $N=N(\epsilon)<\infty$ such that for all $x, n$ :

$$
H_{n+N}(x) \subset B_{n}(x, \epsilon)
$$

${ }^{* *}$ If $f$ is completely discontinuous, then for every $\epsilon>0$ small enough, for all $x$ and $n$ :

$$
B_{n+1}(x) \subset H_{n}(x) .
$$

Proof. Compactness of $[0,1]$ implies that $\max \{\operatorname{diam}(J): J$ is an $n$-homterval $\}$ converges to zero as $n \rightarrow \infty$. In particular, for every $\epsilon>0$, there exists $N<\infty$ such that $H_{n+N}(x) \subset B_{n}(x, \epsilon) .\left({ }^{*}\right)$ is proved.

$\left({ }^{* *}\right)$ is a restatement of Lemma 2.2 .

Hence, to prove the specification property, it is enough to prove the following claim:

2.4 Claim. There exists $D<\infty$ such that for every finite collection of finite itineraries $A^{i} \in P^{n_{i}}, i=1, \ldots, r$, there exists sequences $B^{i} \in P^{D}, i=1, \ldots, r$, such that the infinite, periodic sequence defined by the infinite repetition of the block of length $n_{1}+\cdots+n_{r}+r D$ :

$$
A^{1} B^{1} \ldots A^{r} B^{r}
$$

is the itinerary of some point $x$.

To study this question we shall use Hofbauer's Markov diagram. Define recursively $P_{0}=P$ and $P_{n+1}=\left\{f(A) \cap Z \neq \emptyset: A \in P_{n}, Z \in P\right\}$. The Markov diagram of $f$ is:

$$
\mathcal{M}=\bigcup_{n \geq 0} P_{n}
$$

Elements of $\mathcal{M}$ are open intervals. They are not necessarily disjoint. Their endpoints are points of $C^{+}(f)=\left\{f^{k}(c \pm): k \geq 0, c \in C(f)\right.$ and $\left.\pm \in\{-,+\}\right\}$, i.e., post-critical points. More precisely, $P_{n}=\left\{f^{n}(J): J\right.$ is a $n+1$-homterval $\}$.

$\mathcal{M}$ is countable. It has a natural (oriented) graph structure: $A \rightarrow B$ iff $B=$ $f(A) \cap Z$ for some $Z \in P$. A path on $\mathcal{M}$ is a finite or infinite sequence $\alpha$ such that $\alpha_{k} \rightarrow \alpha_{k+1}$.

For each element $I$ of $\mathcal{M}$ there exists a unique element $\pi(I)$ of $P$ containing it. This map $\pi: \mathcal{M} \rightarrow P$ induces a projection from the set of paths on $\mathcal{M}$ to $\bigcup_{k \geq 1} P^{k}$.

For every finite path $\alpha, A=\pi(\alpha)$ is a finite itinerary which defines a (non-empty) $n$-homterval, if $n$ is the length of $\alpha$. Indeed, writing $H$ for this $n$-homterval, the definition of the graph structure implies that:

$$
\begin{aligned}
\emptyset \neq \alpha_{n-1} & =f\left(\alpha_{n-2} \cap f^{-1}\left(A_{n-1}\right)\right)=\ldots \\
& =f^{n-1}\left(\alpha_{0} \cap f^{-1}\left(A_{1}\right) \cdots \cap f^{-n+1}\left(A_{n-1}\right)\right) \\
& =f^{n-1}\left(\alpha_{0} \cap A_{0} \cap f^{-1}\left(A_{1}\right) \cdots \cap f^{-n+1}\left(A_{n-1}\right)\right) \\
& \subset f^{n-1}\left(A_{0} \cap f^{-1}\left(A_{1}\right) \cdots \cap f^{-n+1}\left(A_{n-1}\right)\right)=f^{n-1}(H) .
\end{aligned}
$$

For infinite paths the situation is slightly more complex: 
2.5 Lemma. There exists a finite exceptional set of periodic itineraries $\mathcal{X}$ such that the following holds:

Every loop on $\mathcal{M}$ (i.e. periodic path) $\alpha$ such that $\pi(\alpha) \notin \mathcal{X}$, defines one point $x \in[0,1]$ with itinerary $\pi(\alpha)$. In particular, $x$ has the same period as $\alpha$.

Proof. To see this, let $A \in P^{\mathbb{N}}$ be the projection of the loop. Consider $\bigcap_{k \geq 0} f^{-k}\left(A_{k}\right)$. If this intersection is not empty, then it is the desired point. Assume it is empty. $\bigcap_{k \geq 0} \overline{A_{0} \cap \cdots \cap f^{-k}\left(A_{k}\right)}$ is not empty, by $\left(^{*}\right)$ and compactness, and therefore is equal to some point $y$. It must be in $\partial\left(A_{0} \cap \cdots \cap f^{-k}\left(A_{k}\right)\right)$ for large $k$, so that the virtual orbit starting from $y \pm$ must go through $C(f)$. It must be periodic like the sequence $A$.

Therefore we can take as the exceptional set $\mathcal{X}$, the set of the itineraries of periodic virtual orbits. It is indeed finite (since $C(f)$ is finite). The lemma is proved.

Let us state some facts about the orbit of an interval. Obviously, if $I$ is a nontrivial interval then $f^{k}(I)$ is the union of a finite number of non-trivial intervals - remember that the critical points have no image under $f$. We will need the following result:

2.6 Proposition (F. Hofbauer [9, corollary, p. 382]). Let $f$ be a topologically transitive p.m.m.

Then for any non-trivial interval $J$,

$$
\bigcup_{k \geq 0} f^{k}(J)=\bigcup_{k=0}^{K} f^{k}(J)=[0,1] \backslash Y
$$

for some $K<\infty, Y \subset[0,1]$ finite, both depending on $J$.

For the sake of completeness we include a proof of this result of F. Hofbauer in Appendix B.

Let us remark that for families satisfying the additional assumption (A4) (in particular the $\beta$-transformations), there is a very simple, direct proof of this fact (see 3.5).

Call any path $\alpha$ such that $\pi(\alpha)=A$ a lift of the itinerary $A$.

It is easy to see that an itinerary $A$ admits a lift $\alpha$ starting from some $\alpha_{0} \in \mathcal{M}$ iff $A$ is the itinerary of an orbit or a virtual orbit starting from the interval $\alpha_{0}$ (for a virtual orbit $x \pm$ this means $] x, x \pm h\left[\subset \alpha_{0}\right.$ for small $h>0$ ).

Though an itinerary may obviously have a lot of lifts on $\mathcal{M}$, we have a kind of "eventual uniqueness" of the lifts of itineraries:

2.7 Lemma (F. Hofbauer [8]). Let $\mathcal{B}$ be the set of the itineraries of the post-critical virtual orbits $\left\{f^{k}(c \pm)\right\}_{k \geq k_{0}},\left(c \in C(f), k_{0} \geq 0\right)$.

Given an infinite itinerary $A \notin \mathcal{B}$ and two paths $\alpha$ and $\beta$ on $\mathcal{M}$ projecting both to $A$, there exists $n<\infty$ such $\alpha_{k}=\beta_{k}$ for all $k \geq n$.

Proof. This lemma follows from $\left(^{*}\right)$ above applied to $\alpha_{k}$ and $\beta_{k}$. As $f$ has no homterval, $\bigcap_{k \geq 0} \overline{f^{-k}\left(A_{k}\right)}$ is a single point. As $A \notin \mathcal{B}$, this point must belong to the open intervals $\alpha_{0}$ and $\beta_{0}$. Hence $A_{0} \cap f^{-1}\left(A_{1}\right) \cap \cdots \cap f^{-k}\left(A_{k}\right) \subset \alpha_{0} \cap \beta_{0}$ for $k$ big enough. But then $\alpha_{k}=\beta_{k}$. The lemma is proved.

Let us show that assumption (S2) implies that one can return to a fixed, finite part of $\mathcal{M}$ in a bounded time: 
2.8 Lemma. There exists $N_{1}<\infty$ such that, starting from any element of $\mathcal{M}$ there exists a path of length at most $N_{1}$ going to an element of $Q_{N_{1}}=\bigcup_{k=0}^{N_{1}} P_{k}$.

Proof. First, one can easily prove that every $J \in \mathcal{M}$ is the homeomorphic image by some iterate $f^{m}$ of $f$, of an interval of the form $] a, f^{k}(b)[$ with $a, b \in C(f)$ (here we disregard the respective order of $a$ and $f^{k}(b)$ ). Therefore $\left|a-f^{k}(b)\right| \geq \alpha$ (the constant defined by (S2)).

Fix $N_{1}<\infty$ such that all $N_{1}$-homtervals have length strictly less than $\alpha$. Therefore there exists $m \leq q<N_{1}$ such that $] a, f^{k}(b)$ [ is in a $q$-homterval but not in a $q+1$-homterval: $\left.f^{i+1}\right] a, f^{k}(b)\left[\right.$ is the unique successor of $\left.f^{i}\right] a, f^{k}(b)[$ for $i=m, \ldots, q-1$ and $\left.f^{q}\right] a, f^{k}(b)[$ has several successors; among them $] f^{q+1} a, c[$ with $c \in C(f)$. But then this is $f^{q+1} H_{q+2}(a \pm)$ (with the sign according to the side of $a$ on which sits $\left.f^{k}(b)\right)$. Hence this successor belongs to $P_{q+1}$ with $q+1 \leq N_{1}$. The lemma is proved.

Recall that the oriented graph $\mathcal{M}$ has a natural pre-order: $A \preceq B$ iff $A=B$ or there exists a path from $A$ to $B$. Corresponding equivalence classes are called irreducible parts. An irreducible part is either (i) trivial: a single element with no loops going through; or (ii) a $\subset$-maximal subset of $\mathcal{M}$ with the property that for any two of its elements $A, B$ there exists a path from $A$ to $B$ to $A$.

Each non-trivial irreducible part has a unique period: the largest integer $p$ such that, for any two elements $A, B$, there exists $k_{0} \in\{0, \ldots, p-1\}$ such that the lengths of the paths from $A$ to $B$ are of the form $k_{0}+m p$ with $m$ an integer. The previous lemma and the topological mixing imply the following properties of the Markov diagram.

2.9 Lemma. $\mathcal{M}$ contains $\mathcal{M}^{\prime}$ such that:

1. $\mathcal{M}^{\prime}$ is a non-trivial irreducible part of $\mathcal{M}$.

2. $\mathcal{M}^{\prime}$ is closed, i.e., all the successors of any element of $\mathcal{M}^{\prime}$ are again elements of $\mathcal{M}^{\prime}$.

3. $\mathcal{M}^{\prime}$ has period 1 .

4. $\cup \mathcal{M}^{\prime}$ is dense in $[0,1]$.

Proof. We use Lemma 2.8 to show that there exists a closed, irreducible part. It is convenient to remark that an irreducible part is closed iff it is $\preceq$-maximal. Now, consider the set of irreducible parts which meet $Q_{N_{1}}$. This set is finite. Hence it admits a $\preceq$-maximal element, $\mathcal{M}^{\prime}$.

Let $A \in \mathcal{M}$ be such that $\mathcal{M}^{\prime} \preceq A$. By Lemma 2.8 , there exists a path from $A$ to some $B \in Q_{N_{1}}$. Hence $\mathcal{M}^{\prime} \preceq B$. But $\mathcal{M}^{\prime}$ is $\preceq$-maximal in $Q_{N_{1}}$. Hence, $B \in \mathcal{M}^{\prime}$. Now $\mathcal{M}^{\prime} \preceq A \preceq \mathcal{M}^{\prime}$ implies, by irreducibility, that $A \in \mathcal{M}^{\prime} . \mathcal{M}^{\prime}$ is closed.

Let $p$ be the period of $\mathcal{M}^{\prime}$ and let $A \in \mathcal{M}_{0}^{\prime}$. Because of topological mixing, $f^{k}(A) \cap A$ has more than three points for large $k$. As $f^{k}(A)$ is the union of nontrivial intervals, this intersection has non-empty interior. Similarly, for large $l$, $f^{l}(A) \cap f^{k}(A) \cap A$ has non-empty interior, so it contains points not in $C^{-}(f) \cup C^{+}(f)$ (which is countable). Consider $x, y \in A$ such that $f^{k}(x)=f^{l}(y) \in A$. $x, y \in$ $A \backslash C^{-}(f)$ define paths $\alpha, \beta$ starting from $A . \iota\left(f^{k}(x)\right) \notin \mathcal{X}$. Applying Lemma 2.7, we see that $\alpha_{k+n}=\beta_{l+n}$ for large $n$. By the definition of the period, $l-k$ must be a multiple of $p$. But $l$ could be chosen freely: $p$ must be equal to 1 .

Finally, we remark that for any interval $A \in \mathcal{M}, f(A)$ is the union of the successors of $A$ in $\mathcal{M}$ and, possibly, of some critical points, which have no image under 
f. Hence, $\bigcup_{k \geq 0} f^{k}\left(\bigcup \mathcal{M}^{\prime}\right) \subset \bigcup \mathcal{M}^{\prime} \cup C(f)$, as $\mathcal{M}^{\prime}$ is a closed graph. As $f$ is topologically mixing this implies that $\bigcup \mathcal{M}^{\prime}$ is dense.

The lemma is proved.

Proof of Sufficiency. It remains to prove Claim 2.4.

Using Proposition 2.6, we see that there exists a finite subset $\mathcal{M}^{\prime \prime} \subset \mathcal{M}^{\prime}$ such that $\bigcup_{I \in \mathcal{M}^{\prime \prime}} I=[0,1] \backslash X_{0}$ (with $X_{0}$ finite).

Let $x \in[0,1]$ and $n \geq 0$. We shall find a path starting in the finite subset $\mathcal{M}^{\prime \prime}$, the beginning of which will project to the $n$-itinerary of $x$ and which return to some fixed element of $\mathcal{M}^{\prime \prime}$ in a fixed amount of time.

By moving $x$ within $H_{n+1}(x)$, we can make sure that it is not in the finite set $X_{0}$. Hence there exists a path $\alpha_{0} \ldots \alpha_{n}$ starting from an element of $\mathcal{M}^{\prime \prime}$ and projecting to the $n$-itinerary of $x$. As $\mathcal{M}^{\prime}$ is closed, we shall always remain in $\mathcal{M}^{\prime}$.

By Lemma 2.8, there exists a path of length at most $N_{1}$ from $\alpha_{n}$ to some element of $Q_{N_{1}} \cap \mathcal{M}^{\prime}$. Let $\mathcal{M}^{\prime \prime \prime} \subset \mathcal{M}^{\prime}$ be a finite set containing at least one predecessor of each element of $\mathcal{M}^{\prime \prime}$. As $Q_{N_{1}} \cap \mathcal{M}^{\prime}$ and $\mathcal{M}^{\prime \prime \prime}$ are finite, the fact that the period of $\mathcal{M}^{\prime}$ is 1 implies that there exists $N_{2}<\infty$ such that for any $I \in Q_{N_{1}} \cap \mathcal{M}^{\prime}$ and $J \in \mathcal{M}^{\prime \prime \prime}$ there exists a path from $I$ to $J$ of any given length greater or equal to $N_{2}$.

Therefore we can complete the previous path $\alpha_{0} \ldots \alpha_{n}$ into a path $\alpha(x, n, \gamma)$ of length $n+N_{1}+N_{2}$ ending at a predecessor of any prescribed element $\gamma$ of $\mathcal{M}^{\prime \prime}$.

To make sure that the path we build defines a point, we make the following remark. As the exceptional set $\mathcal{X}$ of Lemma 2.5 is a finite set of periodic itineraries, we can find a finite itinerary $C$ which does not appear as a subsequence of any itinerary in $\mathcal{X}$. We chose a path $\gamma(\alpha)$ of constant length $N_{3}$, starting from a fixed element $\gamma_{0}$ of $\mathcal{M}^{\prime \prime}$, ending at a predecessor of any prescribed element $\alpha$ of $\mathcal{M}^{\prime \prime}$ and such that the beginning of its projection is the finite itinerary $C$. Given $\left(x_{1}, n_{1}\right), \ldots,\left(x_{r}, n_{r}\right) \in[0,1] \times \mathbb{N}$, we consider the periodic path defined by the repetition of:

$$
\alpha\left(x_{1}, n_{1}, \gamma_{0}\right) \gamma\left(\alpha_{2}\right) \alpha\left(x_{2}, n_{2}, \gamma_{0}\right) \gamma\left(\alpha_{3}\right) \ldots \alpha\left(x_{r}, n_{r}, \gamma_{0}\right) \gamma\left(\alpha_{1}\right)
$$

(here $\alpha_{i}$ is the first symbol of $\alpha\left(x_{i}, n_{i}, \gamma_{0}\right)$ ).

Claim 2.4 is proved and with it the sufficiency of (S1)-(S2).

\section{Proof of the Main Theorem}

Because of the $C^{1}$-smoothness of the p.m.m. family $f$, the initial parameter interval can be written as a countable union of parameter intervals $T$ such that:

(1) $\left\{f_{t}\right\}_{t \in T}$ is uniformly expanding: there exists $\lambda>1$ such that:

$$
\left|\partial_{x} f_{t}(x)\right| \geq \lambda \quad\left(\forall x \in[0,1) \backslash C\left(f_{t}\right), t \in T\right) .
$$

From now on, we consider one such interval. As remarked by M. Benedicks and L. Carleson [1], expansion with respect to $x$ and expansion with respect to $t$ are related by the following computation:

3.1 Lemma. Let $\left(f_{t}\right)_{t \in T}$ be a $C^{1}$ family of p.m.m.'s. We have:

$$
r_{n}(x, t):=\frac{\partial_{t} f_{t}^{n}(x)}{\partial_{x} f_{t}^{n}(x)}=\sum_{k=0}^{n-1} \frac{\partial_{t} f_{t}\left(f_{t}^{k}(x)\right)}{\partial_{x} f_{t}^{k+1}(x)}
$$

for all $x \in[0,1]$ and $n \geq 0$ such that both sides are defined. 
Proof. We have:

$$
\begin{aligned}
\partial_{t} f_{t}^{n+1}(x) & =\left.\partial_{t} f_{t}(y)\right|_{y=f_{t}^{n}(x)}+\left.\partial_{x} f_{t}(y)\right|_{y=f_{t}^{n}(x)} \partial_{t} f_{t}^{n}(x), \\
\partial_{x} f_{t}^{n+1}(x) & =\left.\partial_{x} f_{t}(y)\right|_{y=f_{t}^{n}(x)} \partial_{x} f_{t}^{n}(x) .
\end{aligned}
$$

Set $r_{n+1}=\frac{\left.\partial_{t} f_{t}(y)\right|_{y=f_{t}^{n}(x)}}{\partial_{x} f_{t}^{n+1}(x)}+r_{n}$. Hence:

$$
r_{n+1}=\sum_{k=0}^{n} \frac{\left.\partial_{t} f_{t}(y)\right|_{y=f_{t}^{n}(x)}}{\partial_{x} f_{t}^{n+1}(x)} .
$$

Let $e(t)$ be the non-degenerate endpoint satisfying condition (A2). The derivative with respect to $t$ of $f_{t}^{n}(e(t))$ is:

$$
D_{t}\left[f_{t}^{n}(e(t))\right]=e^{\prime}(t) \partial_{x} f_{t}^{n}(e(t))+\partial_{t} f_{t}^{n}(e(t))
$$

Hence $\sigma(t)=\frac{D_{t}\left[f_{t}^{n}(e(t))\right]}{\partial_{x} f_{t}^{n}(e(t))}$ is equal, by the previous lemma to:

$$
\sigma(t)=e^{\prime}(t)+\sum_{n \geq 0} \frac{\partial_{t} f_{t}\left(f_{t}^{n}(e(t))\right)}{\partial_{x} f_{t}^{n+1}(e(t))}
$$

In general, $\sigma(t)$ has a dense set of discontinuities. But, under the hypothesis of uniform expansion (1), the series is uniformly converging and therefore: the set $\{t \in T: \sigma(t) \neq 0\}$ can be written as the countable union of intervals $T^{\prime}$ on each one of which:

(2) there exists $C<\infty$ such that:

$$
C^{-1} \leq\left|\frac{D_{t}\left[f_{t}^{n}(e(t))\right]}{\partial_{x} f_{t}^{n}(e(t))}\right| \leq C \quad(n \geq 0)
$$

Proof of the density of $T_{\text {coll }}$. We remark that (1) and (2) imply that:

$$
\left|D_{t}\left[f_{t}^{n}(e(t))\right]\right| \geq C^{-1} \lambda^{n}
$$

This implies immediately that the intervals of continuity of $t \mapsto f_{t}^{n}(e(t))$ have their lengths bounded by $C \lambda^{-n}$. But the discontinuities of these functions correspond to values of $t$ such that $f_{t}^{k}(e(t)) \in C\left(f_{t}\right)$ for some $0 \leq k<n$. Hence the set $T_{\text {coll }}$ is dense in $T$ as stated.

As usual, expansion implies a control on distortion:

3.2 Distortion Lemma. Let $\left(f_{t}\right)_{t \in T}$ be a $C^{1+\theta}$ family of p.m.m.'s $(0<\theta<1)$, uniformly expanding and non-degenerate, i.e., satisfying conditions (1) and (2) above.

There exists a uniform bound $K<\infty$ on the distortion of every $F_{n}: t \mapsto$ $f_{t}^{n}(e(t))$, for every $n \geq 0$, on each interval $U \subset T$ on which $F_{n}$ is continuous:

$$
\sup _{t_{1}, t_{2} \in U}\left|\frac{F_{n}^{\prime}\left(t_{1}\right)}{F_{n}^{\prime}\left(t_{2}\right)}\right|<K
$$

This result is of course trivial in the case of a family of piecewise affine maps like the $\beta$-transformations. 
Proof. First we strengthen (2): there exists constants $k_{0}<\infty, C^{\prime}>0$, such that, for all $k_{0} \leq k \leq n$,

$$
\left|D_{x}\left(F_{n} \circ F_{k}^{-1}\right)(x)\right| \geq C^{\prime} \lambda^{n-k} \quad\left(x \in F_{k}(U)\right) .
$$

Indeed, $F_{n} \circ F_{k}^{-1}(x)=f_{t}^{n-k}(x)$ where $t=F_{k}^{-1}(x)$. Taking the derivative:

$$
\begin{aligned}
D_{x}\left(F_{n} \circ F_{k}^{-1}\right)(x) & =\partial_{x} f_{t}^{n-k}(x)+\left(F_{k}^{-1}\right)^{\prime}(x) \partial_{t} f_{t}^{n-k}(x) \\
& =\partial_{x} f_{t}^{n-k}(x)\left[1+\left(F_{k}^{-1}\right)^{\prime}(x) r_{n-k}(x, t)\right] .
\end{aligned}
$$

But $\left|F_{k}^{\prime}\right| \geq C \lambda^{k}$ and, because of Lemma 3.1, the functions $\left\{r_{n}(x, t)\right\}_{n \geq 0}$ are uniformly bounded by a constant $M$ which may be taken to be: $\sup \left|\partial_{t} f_{t}\right| \sum_{m \geq 1} \lambda^{-m}$. Thus,

$$
\left|D_{x}\left(F_{n} \circ F_{k}^{-1}\right)(x)\right| \geq C\left(1-C \lambda^{-k} \cdot M\right) \cdot \lambda^{n-k} .
$$

We get $\left({ }^{* *}\right)$ for $k \geq k_{0}:=[\log C M / \log \lambda]+2$ and $C^{\prime}=\left(1-\lambda^{-1}\right) C$.

As $] F_{k}\left(t_{1}\right), F_{k}\left(t_{2}\right)\left[\right.$ is mapped by $F_{n} \circ F_{k}^{-1}$ to $] F_{n}\left(t_{1}\right), F_{n}\left(t_{2}\right)[\subset] 0,1[,(* *)$ implies:

$$
\left|F_{k}\left(t_{1}\right)-F_{k}\left(t_{2}\right)\right| \leq C^{-1} \lambda^{-(n-k)} \text {. }
$$

But, because of (2), with $C^{ \pm 1}$ standing for numbers in $\left[C^{-1}, C^{1}\right]$,

$$
\left|F_{n}^{\prime}\left(t_{i}\right)\right|=C^{ \pm 1}\left|\partial_{x} f_{t_{i}}^{n}\left(e_{t_{i}}\right)\right|=C^{ \pm 1} \prod_{k=0}^{n-1}\left|\partial_{x} f_{t_{i}}\left(F_{k}\left(e\left(t_{i}\right)\right)\right)\right| .
$$

As $\partial_{x} f_{t}$ is Hölder with exponent $\theta>0$ and is bounded away from zero (by $\lambda$ ), $\log \left|\partial_{x} f_{t}\right|$ is also Hölder with the same exponent. This is true with respect to $x$ as well as with respect to $t$. Hence there exists $C^{\prime \prime}<\infty$ such that:

$$
\begin{aligned}
\log \left|\frac{F_{n}^{\prime}\left(t_{1}\right)}{F_{n}^{\prime}\left(t_{2}\right)}\right| \leq & 2 \log C+\sum_{k=0}^{n-1}|\log | \partial_{x} f_{t_{1}}\left(F_{k}\left(t_{1}\right)\right)|-\log | \partial_{x} f_{t_{1}}\left(F_{k}\left(t_{2}\right)\right)|| \\
& +|\log | \partial_{x} f_{t_{1}}\left(F_{k}\left(t_{2}\right)\right)|-\log | \partial_{x} f_{t_{2}}\left(F_{k}\left(t_{2}\right)\right)|| \\
\leq & 2 \log C+\left(\sum_{k=0}^{n-1} C^{\prime \prime}\left|F_{k}\left(t_{1}\right)-F_{k}\left(t_{2}\right)\right|^{\theta}\right)+n C^{\prime \prime}\left|t_{1}-t_{2}\right|^{\theta} \\
\leq & 2 \log C+C^{\prime \prime} k_{0}+C^{\prime \prime}\left(\sum_{k=k_{0}}^{n-1} C^{-\theta} \lambda^{-(n-k) \theta}\right)+n C^{\prime \prime} C^{\prime-1} \lambda^{-n \theta}
\end{aligned}
$$

From this we deduce the existence of the constant $K<\infty$ as stated.

3.3 Definition. $t \in T$ is $n$-admissible $(n \geq 1)$ if, for all $0 \leq k \leq n$,

$$
\left[F_{k}(t), F_{k}(t) \pm \alpha\left[\cap C\left(f_{t}\right)=\emptyset .\right.\right.
$$

The sign \pm being plus if the wrong side is on the right, minus otherwise.

An interval $U \subset T$ is $n$-admissible $(n \geq 1)$ if the mappings $F_{k}: t \mapsto f_{t}^{k}(e(t))$, $0 \leq k \leq n$, are continuous on $U$ and if every $t \in U$ is $n$-admissible.

We prove the theorem. Let $e=e(t)$ be a critical endpoint. Remark that whether the wrong side of $f_{t}^{n}(e(t))$ is at the left or at the right is determined by the $n$ itinerary of $e$ and is therefore constant on each continuity interval of $F_{n}$. 
Criterium (S2) of Proposition 2.1 implies that $f_{t}$ may have the specification property only if $t$ is $n$-admissible for all $n \geq 0$ and some $\alpha>0$. Hence it is enough to prove that, for every small $\alpha>0$, the set of $n$-admissible $t$, which is a finite union of $n$-admissible intervals, has Lebesgue measure zero. But this follows immediately from the:

3.4 Claim. There exists $\kappa<1$ such that, for all $n \geq 0$, if $U$ is an $n$-admissible interval then there exists $m>n$ such that the union $U^{\prime}$ of $m$-admissible intervals meeting $U$ and hence, included in $U$, is of Lebesgue measure at most $\left|U^{\prime}\right| \leq \kappa \cdot|U|$.

The theorem will follow. Let us prove the claim. Let $U \subset T$ be a non-trivial, $n$-admissible interval. Let $\kappa=1-\alpha / K$.

If $U$ is $(n+p)$-admissible then, according to (3),

$$
\left|F_{n+p}(U)\right| \geq C^{-1} \lambda^{n+p}|U| .
$$

But $\left|F_{n+p}(U)\right| \leq 1$, hence there exists $p<\infty$ maximal with the property that $U$ is $(n+p)$-admissible. Thus $F_{n+p}$ is continuous on $U$ whereas some $t \in T$ are not $(n+p+1)$-admissible: for some discontinuity $d(t), F_{n+p}(t) \in[d(t), d(t) \pm \alpha[$ ( $\operatorname{sign}$ \pm according to wrong side).

For the sake of clarity, we restrict ourselves to the case where there is only one such discontinuity, the general case being similar. By considering $F_{n+p}(t)-d(t)$ instead of $F_{n+p}(t)$, we may assume that $d(t)=d$, a constant: as $\left|F_{n+p}^{\prime}(t)\right| \geq C \lambda^{n+p}$ whereas $d^{\prime}(t)$ stays bounded, this will practically not change the relevant properties of expansion and distortion.

The $t \in U$ which are not $(n+p+1)$-admissible are therefore the ones for which $f_{t}^{n+p}(e) \in\left[d, d+\alpha\left[\right.\right.$ (if the wrong side is, for instance, to the left of $f_{t}^{n}(e(t))$ on $U$ ). If

$$
\mid\left[d, d+\alpha\left[\cap F_{n+p}(U)|<\alpha| F_{n+p}(U) \mid \leq \alpha,\right.\right.
$$

then $\left[d, d+\alpha\left[\right.\right.$ meets $\partial F_{n+p}(U)$ so that $U$ contains only one maximal $(n+p+$ 1)-admissible interval and this interval $V$ satisfies, according to the distortion Lemma 3.2:

$$
|V| \geq\left(1-K \frac{\mid F_{n+p}(U) \cap[d, d+\alpha[\mid}{\left|F_{n+p}(U)\right|}\right)|U| \geq(1-K \alpha)|U|
$$

hence $\left|F_{n+p}(V)\right| \geq C \lambda^{n+p}(1-K \alpha)|U|$. As $\lambda^{p}(1-K \alpha) \geq \lambda^{1 / 2}>1(\alpha>0$ being small and $p \geq 1$ ), it follows that, after $r$ meetings of this kind, the maximal admissible interval is $V_{r}$ and, with obvious notations,

$$
\left|F_{n+p_{1}+\cdots+p_{r}}\left(V_{r}\right)\right| \geq C \lambda^{n+p_{1}+\cdots+p_{r}}(1-K \alpha)^{r}|U| \geq C \lambda^{n+\frac{r}{2}}|U|
$$

and therefore $(*)$ cannot happen an infinite number of times: there exists $m>n$, such that $U$ contains a unique maximal $(m-1)$-admissible subinterval $W$ and $W$ is not $m$-admissible and, with notations as above, $\mid\left[d, d+\alpha\left[\cap F_{m}(W)|\geq \alpha| F_{m}(W) \mid\right.\right.$. Hence, according to the distortion lemma, the part of $W$ mapped into $[d, d+\alpha[$ - which is not $m$-admissible - is a proportion of $W$ at least equal to $K^{-1} \alpha$ and we have, writing $U^{\prime}$ for the set of $m$-admissible parameters in $U$ :

$$
\left|U^{\prime}\right| \leq|W|-\left|\left(F_{m} \mid W\right)^{-1}(] d, d+\alpha[)\right| \leq\left(1-K^{-1} \alpha\right)|W| \leq\left(1-K^{-1} \alpha\right)|U| .
$$

The claim, and hence the theorem, is proved. 
Proof of the corollary of the Main Theorem.

3.5 Lemma. (A3) +(A4) imply topological mixing.

If an interval does not contain a discontinuity in its interior, $f_{t}$ multiplies its length by $\lambda>1$. It follows that if $I$ is a non-trivial interval, then there exists $n(I)<\infty$ such that $f_{t}^{n(I)}(I)$ contains a discontinuity $c$ in its interior and, as $f_{t}(c+)=0, f_{t}^{n(I)+1}(I)$ contains an interval of the form $] 0, \epsilon[$ for $\epsilon>0$ small enough. But $\left.f_{t}^{k}(] 0, \epsilon[)=\right] 0,1\left[\right.$ as soon as $k>\frac{|\log \epsilon|}{\log \lambda}$. The lemma is proved.

Let $t \in T_{\text {coll }}: f_{t}^{n}(c \pm)=d$ for some $c, d \in C\left(f_{t}\right), n \geq 1$. We now use the equations in (A4). First, the sign \pm is necessarily the minus sign. Second, $f_{t}$ is piecewise increasing. Hence, $f_{t}^{n}(c-)=d-$. Now $f^{n}(d-)=f^{n-1}(1-)=f^{n}(c-)=d-$ : the virtual orbit defined by $d-$ is periodic. Therefore:

$$
C^{+}(f)=\left\{0, d_{1}, \ldots, d_{N-1}, 1-, f_{t}(1-), \ldots, f_{t}^{n-2}(1-)\right\} .
$$

In particular, it is finite. This implies that (S2) is satisfied.

Hence the criterium 2.1 implies that $f_{t}$ has the specification property for all $t \in T_{\text {coll }}$. We have seen (Main Theorem) that $T_{\text {coll }}$ is dense. The corollary is proved.

\section{Application to families like $\left(T_{\beta}\right)_{\beta>1}$}

First let us remark that everything we are going to do for the $\beta$-transformations is true for expanding maps modulo 1 (see the introduction). Let us remark also that the $\beta$-transformations define a $C^{\infty}$ family of p.m.m.'s. Conditions (A1), (A3) and (A4) are obviously satisfied. We have to check condition (A2). We will use the following notion, which obviously includes the $\beta$-transformations as a special case:

4.1 Definition. A family of p.m.m.'s is said to be monotonic if, for $i=1, \ldots, N$.

1. $x \mapsto f_{i}(t, x)$ is strictly increasing, for fixed $t \in T$.

2. $t \mapsto f_{i}(t, x)$ is non-decreasing, for fixed $x \in[0,1]$.

We assume, in addition that there exists an endpoint $e(t)=d_{i}(t) \pm$, an integer $K$ such that the map $t \mapsto f_{t}^{K}(e(t))$ is piecewise strictly increasing.

Let us check that a monotonic p.m.m. family satisfies the non-degeneracy condition (A2). The partial sums in (A2) are: $\sigma_{n}(t)=e^{\prime}(t)+\sum_{k=0}^{n-1} \frac{\partial_{t} f_{t}\left(f_{t}^{k}(e(t))\right)}{\partial_{x} f_{t}^{k+1}(e(t))}$. By Lemma 3.1, $\sigma_{n}(t)=\frac{D_{t}\left[f_{t}^{n}(e(t))\right]}{\partial_{x} f_{t}^{n}(e(t))}$. Hence, introducing $h(t)=f^{K}(e(t))$ and using again Lemma 3.1:

$$
\begin{aligned}
\sigma_{n+K}(t) & =\frac{D_{t}\left[f_{t}^{n}(h(t))\right]}{\partial_{x} f_{t}^{n}(h(t))} \cdot \frac{1}{\partial_{x} f_{t}^{K}(e(t))} \\
& =\left(h(t)^{\prime}+\sum_{k=0}^{n-1} \frac{\partial_{t} f_{t}\left(f_{t}^{k}(h(t))\right)}{\partial_{x} f_{t}^{k+1}(h(t))}\right) \cdot \frac{1}{\partial_{x} f_{t}^{K}(e(t))} .
\end{aligned}
$$

But $\partial_{x} f_{t}^{K}(e(t))$ and $h^{\prime}(t)$ are positive and independent of $n$. The first factor is bounded from below by $h^{\prime}(t)>0$. Hence $\sigma_{n}(t)$ cannot converge to zero: condition (A2) is satisfied.

The Main Theorem therefore applies to $\beta$-transformations with $e(t)=\beta^{-1}-$, $K=2$ : we have the stated counterexample. 
4.2 Remark. Because of the first condition of monotonicity $f_{t}^{n}\left(H_{n+1}(e)\right)$ is on the same side of $f_{t}^{n}(e)$ for all $n \geq 0$. The right endpoints $c+(c \in C(f))$ are mapped to the fixed point 0 : they all satisfy condition (S2). The left endpoints $c-$ are mapped to 1 and $f_{t}^{n}(c-)$ is always the right endpoint of $f_{t}^{n} H_{n+1}(e)$. Hence, if the left endpoint of the interval $f_{t}^{n} H_{n+1}(e)$ is a critical point $\left(f_{t}^{n} H_{n+1}(e)=\right] d_{i}+, f_{t}^{n}(e)[)$, then its image is $\left.f_{t}\left(f_{t}^{n} H_{n+1}(e)\right)=\right] 0, f_{t}^{n+1}(e)[$.

Therefore condition (S2) is equivalent to:

$$
\inf \left\{f_{t}^{n}(1-)>0: n \geq 0\right\}>0
$$

\section{Appendix A. A proof of Blokh's theorem}

We give an independent proof of Theorem 1.1 of A.M. Blokh [3]. We remark that A.M. Blokh has recently published a proof, as a chapter of his monograph [4]. Let $f:[0,1] \rightarrow[0,1]$ be a continuous, topologically mixing map. We do not assume that it is piecewise monotonic. We remark that in that particular case one can give a proof based on Hofbauer's Markov diagram in the spirit of [10]. It uses the fact that, if an interval is split by a critical point into two sub-intervals such that one is very small, one can avoid the small interval by using the map which associates to each point in the neighborhood of an extremum the other which has the same image. But this proof is both more involved and less general than the one we give here. The obvious but fundamental fact about $f$ is that the image of an interval is an interval (the so called "Darboux property"). We shall use it constantly without reference. The following lemma deals with the fact that it is not always true that for any non-trivial interval $I, f^{N}(I)=[0,1]$ for $N$ big enough:

A.1 Lemma. Let $f:[0,1] \rightarrow[0,1]$ be an arbitrary continuous, topologically mixing map.

For every $\alpha, \epsilon>0$, there exists $N(\alpha, \epsilon)<\infty$ and $\delta(\epsilon)>0$ such that, for all $x, y \in[0,1]$, all $n \geq 0$, there exists $z(y, \epsilon) \in B_{n}(y, \epsilon)$-so that the Bowen ball at $z$ is nearly the same as the Bowen ball at $y$ - such that:

$$
f^{N} B(x, \alpha) \supset B(z, \delta) \cap[0,1] .
$$

Proof (of the lemma). Topological mixing and compactness imply that, for every $\alpha, \delta>0$ there exists $N_{1}(\alpha, \delta)<\infty$ such that:

$$
f^{n} B(x, \alpha) \supset[\delta, 1-\delta] \quad\left(\text { for all } x \in[0,1], n \geq N_{1}\right) .
$$

If there exists $a \in] 0,1\left[\right.$ such that $f^{i}(a)=0(i \geq 0$, in fact one can assume $i=1,2)$, we say that 0 is accessible with interior pre-image $a$; likewise for 1 .

If 0 and 1 are accessible with respective interior pre-images $a$ and $b$, then the preceding property implies that $f^{n} B(x, \alpha)=[0,1]$ for $n \geq N_{1}(\alpha, d(\{a, b\},\{0,1\}))+$ 2. Hence just take $N=N_{1}+2, z(y, \epsilon)=y, \delta(\epsilon)=1$.

Assume, for instance, that 1 is accessible but not 0 (the other cases are similar). As the topological mixing implies that $f([0,1])=[0,1]$, necessarily $f^{-1}(0)=\{0\}: 0$ is a fixed point. We have the following fact (which we state in a form also suitable for the p.m.m. case):

A.2 Lemma. Let $f:[0,1] \backslash D \rightarrow[0,1]$ be continuous, topologically mixing. Assume that $D \subset] 0,1[$ is finite and that $f$ has a left and a right limit at each point of $D$. Then:

If the following hold: 
(i) for $h>0$ small enough, $f \mid] x_{0}, x_{0}+h[$ is continuous;

(ii) $f\left(x_{0}+\right)=x_{0}+$;

(iii) $f(y \pm) \neq x_{0}+$ for all $y \pm \neq x_{0}+$.

Then fixed points accumulate on $x_{0}+$ (i.e., they accumulate to the right of $x_{0}$ ).

Proof. If ] $x_{0}, x_{0}+h$ [ did not contain any fixed point, $t \mapsto f(t)-t$ would have fixed sign so that one of the following statements would hold:

1. every point of this interval would converge to $x_{0}$ (if $f(t)<t$ );

2. $\left.f^{k}(] x_{0}+u, f\left(x_{0}+u\right)[) \cap\right] x_{0}+u, f\left(x_{0}+u\right)[=\emptyset$ for $k \geq 1(u>0$ fixed, small enough) (if $f(t)>t$ );

(for the last statement remark that $\left.f\left(\left[0, x_{0}[\cup] x_{0}+u, 1\right]\right) \cap\right] x_{0}, x_{0}+u[=\emptyset$ by the last assumption of the lemma).

Both statements contradict topological mixing. Hence $] x_{0}, x_{0}+h[$ contains at least one fixed point. But we can reduce $h>0$ at will. The lemma is proved.

This lemma (with $x_{0}=0+$ ) implies that we may choose a fixed point $p$ distinct from 0 but close to it. We choose it so close that $f^{i}([0, p]) \subset[0, \epsilon / 3[$ for $i=0,1,2$.

Let $\delta=p / 2$ and $\delta^{\prime}=\min (p / 2, d(a,\{0,1\}))$ (with $a$ an interior pre-image of 1 ). Notice that $\delta$ depends only on $\epsilon$. Let $N=N_{1}\left(\alpha, \delta^{\prime}\right)+2$, so that $f^{N} B(x, \alpha) \supset[p / 2,1]$ for every $x \in[0,1]$. Let $y \in[0,1]$. We now have to find $z=z(y, \epsilon) \in B_{n}(y, \epsilon) \cap[p, 1]$.

Let $m=\min \left\{k \geq 0: f^{k}(y) \in[p, 1]\right\}$. If $m=\infty$ then take $z=p$. If $m=0$, take $z=y$. Assume $0<m<\infty$. Remark that $f^{k}(y) \in[0, \epsilon / 3[$ for $k=0, \ldots, m-1$ and also $k=m$.

Topological mixing implies that, for every $0<\beta<\epsilon / 3$, there exists $K(\beta)<$ $\infty$ such that $f^{K}(] p, p+\beta[) \ni f^{m}(y)$. Assume that $K$ is minimal, so that $f^{k}(] p, p+\beta[) \subset[0, \epsilon / 3]$ for $k=0, \ldots, K-1$. By choosing $\beta$ small enough, we may ensure $K \geq m$. Fix $z \in f^{K-m}(] p, p+\beta[)$ a pre-image by $f^{m}$ of $f^{m}(y)$. We see that $z=z(y, \epsilon) \in B_{n}(y, \epsilon)$.

Write $B_{n}^{\prime}(x, \epsilon)$ for the connected component of $B_{n}(x, \epsilon)$ containing $x$.

A.3 Lemma. For every $0<\epsilon<1 / 2, \delta>0$ there exists $N_{2}(\epsilon, \delta)<\infty$ such that:

$$
B_{n}^{\prime}(x, \epsilon) \subset B(x, \delta) \quad\left(\text { for all } x \in[0,1], n \geq N_{2}\right) .
$$

Proof. If this lemma was not true, one could find, by compactness, an $x \in[0,1]$, an $\epsilon>0$ such that $B=\bigcap_{n \geq 1} B_{n}^{\prime}(x, \epsilon)$ would be a non-trivial interval. But $\operatorname{diam}\left(f^{n}(B)\right) \leq 2 \epsilon<1$ for all $n \geq 0$, contradicting topological mixing. The lemma is proved.

Remark that this statement with $B_{n}(x, \epsilon)$ instead of $B_{n}^{\prime}(x, \epsilon)$ is clearly false.

A.4 Lemma. Let $f:[0,1] \rightarrow[0,1]$ be an arbitrary continuous, topologically mixing map. If, for every $\epsilon>0$ :

$$
\alpha(\epsilon)=\inf _{\substack{x \in[0,1] \\ n \geq 0}} \operatorname{diam}\left(f^{n} B_{n}^{\prime}(x, \epsilon)\right)>0
$$

then $f$ has the specification property.

Proof. Fix $\epsilon>0$. Lemma A.1 defines $\delta(\epsilon / 2)>0$. Let $\alpha=\alpha(\epsilon / 2)>0$. Fix $N=N_{1}(\alpha, \epsilon / 2)$ according to Lemma A.1 and $N_{2}(\epsilon / 2, \delta)$ according to Lemma A.3.

Now, let $\left(y_{1}, d_{1}\right), \ldots,\left(y_{r}, d_{r}\right) \in[0,1] \times \mathbb{N}$. We build the periodic point required by the definition of the specification property. 
Let $z_{i}\left(y_{i}, \epsilon / 2\right) \in B_{d_{i}+N_{2}}\left(y_{i}, \epsilon / 2\right)$ be defined as in Lemma A.1. Let:

$$
J(i)=B_{d_{i}+N_{2}}^{\prime}\left(z_{i}, \epsilon / 2\right) \subset B_{d_{i}+N_{2}}\left(y_{i}, \epsilon\right) .
$$

$f^{d_{i}+N_{2}} B_{d_{i}+N_{2}}^{\prime}\left(z_{i}, \epsilon / 2\right)$ is an interval with diameter at least $\alpha$. By the definition of $N_{2}, J(j) \subset B\left(z_{j}, \delta\right)$. Hence, $f^{d_{i}+N_{2}+N}(J(i)) \supset J(j)$, in particular for $j=i+1$ $(j=1$ if $i=r)$.

Let us remark that, if $[a, b] \subset f([c, d])$ then $[a, b]=f\left(\left[c^{\prime}, d^{\prime}\right]\right)$ for some subinterval $\left[c^{\prime}, d^{\prime}\right] \subset[c, d]$. Thus, by an easy recurrence, we build an interval $K$ such that, letting $D=N_{2}+N, f^{d_{1}+\cdots+d_{s-1}+(s-1) D}(K) \subset J(s)$ for $1 \leq s \leq r$ and $f^{d_{1}+\cdots+d_{r}+r D}(K) \supset$ $K$. Hence there exists a periodic point $z \in K$ as requested. The specification property is proved.

Proof of Blokh's theorem. We only have to check the hypothesis of the previous lemma.

First remark: for each $\epsilon>0$, we have, using compactness and topological mixing, a "no-contraction principle":

$$
\beta(\epsilon)=\inf _{I, n \geq 0} \operatorname{diam}\left(f^{n}(I)\right)>0 .
$$

( $I$ ranging over the intervals with diameter at least $\epsilon$ ).

Second remark: if $f^{n+1} B_{n+1}^{\prime}(x, \epsilon) \neq f\left(f^{n} B_{n}^{\prime}(x, \epsilon)\right)$ it is because $B_{n}^{\prime}(x, \epsilon)$ contains points separated by $f^{n+1}$ from $f^{n+1}(x)$ by a distance greater than $\epsilon$. Hence the interval $f^{n+1} B_{n+1}^{\prime}(x, \epsilon)$ has diameter at least $\epsilon$.

An immediate recurrence using both remarks shows that $\operatorname{diam}\left(f^{n} B_{n}^{\prime}(x, \epsilon)\right)$ do not get smaller than $\beta(\epsilon)>0$. Hence, by the previous lemma, the specification property holds. Blokh's theorem is proved.

\section{Appendix B. Proof of Proposition 2.6}

$f:[0,1] \backslash C(f) \rightarrow[0,1]$ is a topologically transitive p.m.m. In particular $f$ has no homtervals. Let $I$ be a non-trivial interval. Set $S=\bigcup_{k \geq 0} f^{k}(I)$ and $S_{n}=\bigcup_{k=0}^{n} f^{k}(I)$. We have to prove that $S$ is a finite union of intervals and that $S=S_{n}$ for finite $n$. It will imply that $S=[0,1] \backslash Y$ with $Y$ a finite set of points.

B.1 Lemma (F. Hofbauer [8]). Let $f:[0,1] \backslash C(f) \rightarrow[0,1]$ be a p.m.m. without homtervals. Then $S$ is a finite union of intervals.

Proof. For each endpoint $c \pm$ of $P$, let $K(c \pm, n)$ be the connected component of $S_{n} \backslash C(f)$ containing $] c, c \pm h[(h>0$ small enough $)$ or the empty set, if there is no such component. If $\left[c, c \pm h\left[\subset S_{n}(h>0\right.\right.$ small enough) then add $c$ to $K(c \pm, n)$. Finally let $K(c \pm)=\bigcup_{n \geq 0} K(c \pm, n)$.

Let $J$ be a non-trivial interval or the empty set. Define $n(J)=0$ if $J=\emptyset$, otherwise let $n(J)$ be the smallest integer $k \geq 0$ such that $f^{k}(J)$ meets $C(f)$. By assumption $n(J)$ is always finite.

Let $A$ be the following union:

$$
\bigcup_{k=0}^{n(I)-1} f^{k}(I) \cup \bigcup_{e} \bigcup_{k=0}^{n(K(e))-1} f^{k}(K(e)) .
$$

(e ranges over the endpoints of $P$.)

Clearly $I \subset A \subset S$ and $f(A) \subset S$. To prove that $S=A$ (a finite union of intervals) it is enough to see that $f(A) \subset A$. 
Consider $f^{k}(K(e))$. If $k<n(K(e))-1$ then $f^{k+1}(K(e)) \subset A$ by definition $\left(^{*}\right)$. Assume $k=n(K(e))-1$. Then $f^{k+1}(K(e))$ meets $C(f)$. As $f^{k+1}(K(e)) \subset f(A) \subset$ $S, f^{k+1}(K(e)) \subset \bigcup_{c \pm} K(c \pm)$ with $c \pm$ ranging over the endpoints of $P$ such that $c \pm \in f^{k+1}(K(e))$ (i.e., $] c, c \pm h\left[\subset f^{k+1}(K(e))\right)$. Hence $f^{k+1}(K(e)) \subset A$. The case of the $f^{k}(I)$ is similar. We have proved that $f(A) \subset A$. Thus $S=A$.

We assume that the union $(*)$ above is a disjoint union (if not, consider:

$$
A^{\prime}=\bigcup_{k=0}^{n(I)-1} f^{k}(I) \times\{(I, k)\} \cup \bigcup_{e}^{n(K(e))-1} \bigcup_{k=0}^{k} f^{k}(K(e)) \times\{(K(e), k)\}
$$

and define $f: A^{\prime} \rightarrow A^{\prime}$ in the obvious way).

We say that $x \pm$ is an endpoint of $S$ (and write $x \pm \in S$ ) if $x \in \partial S$ and $] x, x \pm h[\subset S$ (for small $h>0$ ).

Claim. Every endpoint of $S$ is an endpoint of $S_{n}$ for some $n<\infty$.

Using the disjointness assumption, it is clear that the claim implies that $S=S_{n}$ for large $n$. Let us prove the claim.

Let $X$ be the set of endpoints of $S$ which are not endpoints of $S_{n}$, however large is $n$. $X$ is a finite set. Let $x \pm \in X$. Points close to $x \pm$ are the image by $f$ of points of $S$. Hence, there exists an endpoint $y \mp$ such that $f(y \mp)=x \pm$. If $y \mp$ was a critical point, it would be an endpoint of some $S_{n}$. If $y \mp$ was an endpoint of $S_{n}$, $x \pm$ would be an endpoint of $S_{n+1}$ so that $y \mp$ must be in $X$. From this we deduce that $X$ is a finite union of (possibly virtual) periodic orbits. By replacing $f$ with an iterate $f^{q}$ we may assume that the inacessible endpoints $x \in X$ are fixed by $f$.

Let $x \pm \in X . f$ is piecewise monotonic: we can apply Lemma A.2 to each $x \pm$. Hence, fixed points accumulate on $x \pm$. But if $p$ is a fixed point close enough to $x \pm$ then $f$ is monotonic on $[x \pm, p]$. Hence this interval is invariant. It must be a homterval. As $f$ has no homtervals, $X$ must be empty. The proposition is proved.

\section{REFERENCES}

[1] M. Benedicks and L. Carleson, The dynamics of the Hénon map, Ann. Math. 133 (1991), 73-169. MR 92d:58116

[2] A. Bertrand-Mathis, Développement en base $\theta$, Bull. Soc. Math. France 114 (1986), 271-323. MR 86d:54060

[3] A.M. Blokh, Decomposition of dynamical systems on an interval, Russ. Math. Surv. 38 (1983), 133-134.

[4] The spectral decomposition for one-dimensional maps, Dynamics Reported (Jones et al., eds.), vol. 4, 1995, (new series). MR 96e:58087

[5] R.Bowen, Entropy for group endomorphisms and homogeneous spaces, Trans. Amer. Math. Soc. 153 (1971), 401-414. MR 43:469

[6] M. Denker, C. Grillenberger, K. Sigmund, Ergodic theory on compact spaces, LNM 527, Springer-Verlag, 1976. MR 56:15879

[7] F. Hofbauer, On intrinsic ergodicity of piecewise monotonic transformations with positive entropy, Israel J. Math. I 34 (1979), 213-237; II, vol. 38, 1981, pp. 107-115. MR 82c:28039a; MR 82c:28039b

[8] - The structure of piecewise monotonic transformations, Ergod. Th. \& Dynam. Sys. 1 (1981), 159-178. MR 83k:58065

[9] _ Piecewise invertible dynamical systems, Prob. Th. Rel. Fields 72 (1986), 359-386. MR 87k:58126

[10] Generic properties of invariant measures for continuous piecewise monotonic transformations, Monat. Math. 106 (1988), 301-312. MR 90b:28018 
[11] F. Hofbauer, G. Keller, Ergodic properties of invariant measures for piecewise monotonic transformations, Math. Z. 180 (1982), 119-140. MR 83h:28029

[12] P. Walters, Equilibrium states for $\beta$-transformations and related transformations, Math. Z. 159 (1978), 65-88. MR 57:6370

Université Paris-Sud, BÂt. 425, 91405 Orsay, France

Current address: Université de Bourgogne, Lab. de Topologie, B.P. 400, 21011 Dijon Cedex, France

E-mail address: jerome.buzzi@u-bourgogne.fr 